\title{
Macular corneal dystrophy and associated corneal thinning
}

L Dudakova', M Palos², M Svobodova²,

J Bydzovsky², L Huna², K Jirsova' ${ }^{1}$, AJ Hardcastle ${ }^{3}$,

SJ Tuft ${ }^{3,4}$ and P Liskova ${ }^{1,2}$

\begin{abstract}
Purpose To identify the molecular genetic cause of macular corneal dystrophy (MCD) in four probands, and characterize phenotypic similarities between MCD and keratoconus. Methods We performed ophthalmological examination, Scheimpflug imaging (Pentacam, Oculus Inc.), histopathological examination of excised corneal buttons, and direct sequencing of the CHST6 coding region.

Results Pentacam measurements were taken in six eyes of three probands. All showed diffuse corneal thinning with paracentral steepening of the anterior corneal surface that was graded as keratoconus by the integrated software, but without associated ectasia of the posterior corneal surface or regional thinning. Homozygous or compound heterozygous CHST6 mutations were identified in all cases, including two novel mutations, c.13C $>$ T; p.(Arg5Cys) and c.289C $>$ T; p.(Arg97Cys).

Discussion Localized elevation of the anterior corneal curvature can occur in MCD in the absence of other features of keratoconus. The identification of a further two Czech probands with the compound allele c. [484C $>G ; 599 \mathrm{~T}>\mathrm{G}]$ supports the enrichment of this allele in the study population.
\end{abstract}

Eye (2014) 28, 1201-1205; doi:10.1038/eye.2014.164; published online 1 August 2014

\section{Introduction}

Macular corneal dystrophy (MCD, OMIM \#217800) is an autosomal recessive condition characterized by gray-white stromal opacities with indistinct borders, and haze of the intervening stroma. ${ }^{1}$ Corneal thinning is an important clinical feature of MCD. ${ }^{2} \mathrm{MCD}$ is caused by mutations within the CHST6 gene and is a rare disorder. ${ }^{1}$ CHST6 encodes the enzyme carbohydrate ( $\mathrm{N}$-acetylglucosamine 6-O) sulfotransferase 6 that catalyzes the transfer of a sulfate group to the GlcNAc residues of keratan sulfate, and disease-causing mutations result in abnormal deposition of glycosaminoglycans in corneal stroma, keratocytes, Descemet membrane, and the corneal endothelium. ${ }^{1,3}$

Keratoconus is a complex disorder characterized by bilateral thinning and ectasia of the cornea. It has a strong genetic component and the prevalence is estimated to be between 29 and 229 per 100000 depending on the population studied..$^{4,5}$ Corneal ectasia and thinning, hallmarks of keratoconus, have also been reported in patients with other corneal dystrophies, which has prompted a debate as to whether there could be a common etiology in some affected individuals. ${ }^{6,7}$

We therefore screened CHST6 for mutations in patients diagnosed with MCD and used Scheimpflug imaging to look for features of keratoconus. Excised corneal buttons were examined when available.

\section{Materials and methods}

The research was conducted in accordance with the Declaration of Helsinki and institutional ethical committee approval was obtained. We examined four previously unreported probands (three females and one male) diagnosed with MCD as well as one previously genotyped individual. ${ }^{8}$ Both parents of probands 1, 3, 4, 5 were of white Czech origin, while proband 2 was the child of Moroccan and white Czech parents. Pentacam (Oculus Inc., Wetzlar, Germany) analysis with the automated keratoconus detection software was performed in individuals 1, 2, and 5. Individual 4 had bilateral grafts and individual 3 had such advanced disease that image capture was impossible. Only the anterior surface of the
${ }^{1}$ Laboratory of the Biology and Pathology of the Eye, Institute of Inherited Metabolic Disorders, First Faculty of Medicine, Charles University in Prague and General University Hospital in Prague, Prague, Czech Republic

${ }^{2}$ Department of Ophthalmology, First Faculty of Medicine, Charles University in Prague and General University Hospital in Prague, Prague, Czech Republic

${ }^{3}$ Department of Ocular Biology and Therapeutics, UCL Institute of

Ophthalmology, London, UK

${ }^{4}$ Corneal Service, Moorfields Eye Hospital NHS Foundation Trust, London, UK

Correspondence: P Liskova, Laboratory of the Biology and Pathology of the Eye, Institute of Inherited Metabolic Disorders; First Faculty of Medicine, Charles University in Prague and General University Hospital in Prague, Ke Karlovu 2, 12808 Prague, Czech Republic

Tel/Fax: +420 224967 139; E-mail: petra.liskova@ If1.cuni.cz

Received: 2 December 2013 Accepted in revised form: 31 May 2014 Published online: 1 August 2014 
right cornea of individuals 1 and 5 could be reliably evaluated. Measurements were compared with an individual with keratoconus without any other corneal disorder.

DNA of the cases and available first degree relatives was extracted from venous blood samples using Gentra Puregene Blood Kit (Qiagen, Hilden, Germany) according to the manufacturer's protocol. Coding exons of CHST6 were amplified and sequenced as previously described ${ }^{8,9}$ NCBI NM_021615.4 was used as the reference sequence. Mutation description followed current recommendations of Human Genome Variation Society (HGVS) (http://www.hgvs.org/mutnomen/). ${ }^{10}$ The 1000 Genomes dataset (http://browser.1000genomes. org/index.html) and the Exome Variant Server (EVS, http://evs.gs.washington.edu/EVS/) were examined to determine the frequency of previously unreported sequence variants identified. Potential pathogenicity of missense variants was evaluated using PolyPhen2,11 MutPred, ${ }^{12}$ and SNPs\&GO algorithms. ${ }^{13}$

Corneal buttons obtained after right penetrating keratoplasties of probands 2, 3, and 4 were dissected, snap frozen in liquid nitrogen, and embedded in Optimal Cutting Temperature Compound. Tissue was cryosectioned at a thickness of $7 \mu \mathrm{m}$ and stained with haematoxylin-eosin and Alcian blue for morphological assessment by light microscopy.

\section{Results}

Clinical characteristics and observed genotypes are shown in Table 1. Segregation of the identified mutations in available first degree relatives of probands 1,2, and 4 confirmed that each mutation has been located on a separate chromosome. Proband 3 was the only person studied within the family. The topography data is summarized in Table 2. The family history for MCD was negative in all cases, which is consistent with an autosomal recessive mode of inheritance. Slit-lamp biomicroscopy showed typical changes of MCD, gray-white stromal opacities with indistinct borders and haze of the intervening stroma, without features of keratoconus in all cases.

In all of the six eyes analyzed, a keratoconus pattern could be distinguished on anterior sagittal curvature maps. Examination of the posterior elevation maps using best fit sphere, posterior sagittal curvature, pachymetry maps, and corneal thickness spatial profiles showed diffuse thinning without focal ectasia (Figures 1a and b), which was supported by manual inspection of the

Table 1 Clinical data and genotypes of probands with macular corneal dystrophy

\begin{tabular}{|c|c|c|c|c|c|c|c|}
\hline $\begin{array}{l}\text { Case } \\
\text { no/gender }\end{array}$ & $\begin{array}{c}\text { Subjective age } \\
\text { of onset }(y)\end{array}$ & $\begin{array}{c}\text { Age at } \\
\text { examination } \\
(y)\end{array}$ & \multicolumn{2}{|c|}{$\begin{array}{c}\text { BCVA } \\
\text { prior to } \\
P K\end{array}$} & $\begin{array}{c}\text { Age at } \\
\text { PK }\end{array}$ & CHST6 genotypes & Mutations at protein level \\
\hline $1 / \mathrm{F}$ & 15 & 18 & 0.33 & 0.66 & $19 \mathrm{NP}$ & c. [484C > G; 599T > G];[484C > G; 599T > G] & p.[(Arg162Gly; Leu200Arg)];[(Arg162Gly; Leu200Arg)] \\
\hline $2 / \mathrm{F}$ & 15 & 37 & 0.25 & 0.5 & $37 \mathrm{NP}$ & c. $[1 \mathrm{~A}>\mathrm{T}] ;[484 \mathrm{C}>\mathrm{G} ; 599 \mathrm{~T}>\mathrm{G}]$ & p.[Met1?];[(Arg162Gly; Leu200Arg)] \\
\hline $3 / \mathrm{F}$ & 26 & 32 & 0.33 & 0.33 & 3233 & c. $[289 \mathrm{C}>\mathrm{T}(;) 1046 \mathrm{G}>\mathrm{A}]$ & p.[(Arg97Cys(;)Cys349Tyr)] \\
\hline $4 / \mathrm{M}$ & 26 & 30 & 0.33 & 0.5 & $30 \quad 33$ & c. $[13 \mathrm{C}>\mathrm{T}] ;[1046 \mathrm{G}>\mathrm{A}]$ & p.[(Arg5Cys)];[(Cys349Tyr)] \\
\hline $5 / F^{a}$ & 16 & 18 & 0.66 & 0.66 & NP NP & c.[484C > G; 599T > G];[?] & p.[(Arg162Gly; Leu200Arg)];[?] \\
\hline
\end{tabular}

Abbreviations: BCVA, best corrected visual acuity; LE, left eye; NP, not performed; PK, penetrating keratoplasty; RE, right eye; y, year.

${ }^{a}$ Molecular genetic findings in case 5 have been previously reported. ${ }^{8}$

In case 3 segregation analysis of mutations in the family was not performed therefore a possibility remains that there is a deletion of the second allele as delineated by the HGVS nomenclature with (;). In case 5, screened previously for CHST6 coding sequence variants, mutation in the second allele has not been identified as delineated by the HGVS nomenclature with [?].

Table 2 Pentacam measurements of six corneas with macular corneal dystrophy

\begin{tabular}{lcrrcccl}
\hline Case no/eye & $K 1(D)$ & $K 2(D)$ & $T K C^{\mathrm{a}}$ & $C C T(\mu \mathrm{m})$ & Thinnest $(\mu \mathrm{m})$ & Apex $(\mu \mathrm{m})$ & $\begin{array}{l}\text { Pattern and localization of steepening } \\
\text { on anterior sagittal maps }\end{array}$ \\
\hline 1/RE & 50.8 & 55.4 & $2-3$ & 420 & 371 & 422 & Central and inferotemporal \\
1/LE & 46.5 & 52.9 & 2 & 375 & 335 & 374 & Inferior (asymmetric bow tie with skewed radial axes) \\
2/RE & 42.1 & 46.2 & $1-2$ & 438 & 428 & 436 & Inferotemporal \\
2/LE & 43.4 & 45.2 & 1 & 427 & 416 & 425 & Inferotemporal \\
5/RE & 42.3 & 43.8 & 2 & 409 & 396 & 399 & Inferotemporal \\
5/LE & 45.6 & 47.7 & $2-3$ & 418 & 382 & 419 & Inferotemporal \\
\hline
\end{tabular}

Abbreviations: CCT, central corneal thickness; D, diopter; K1, flat keratometry; K2, steep keratometry; LE, left eye; RE, right eye; TKC, topographic keratoconus classification.

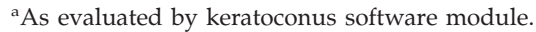



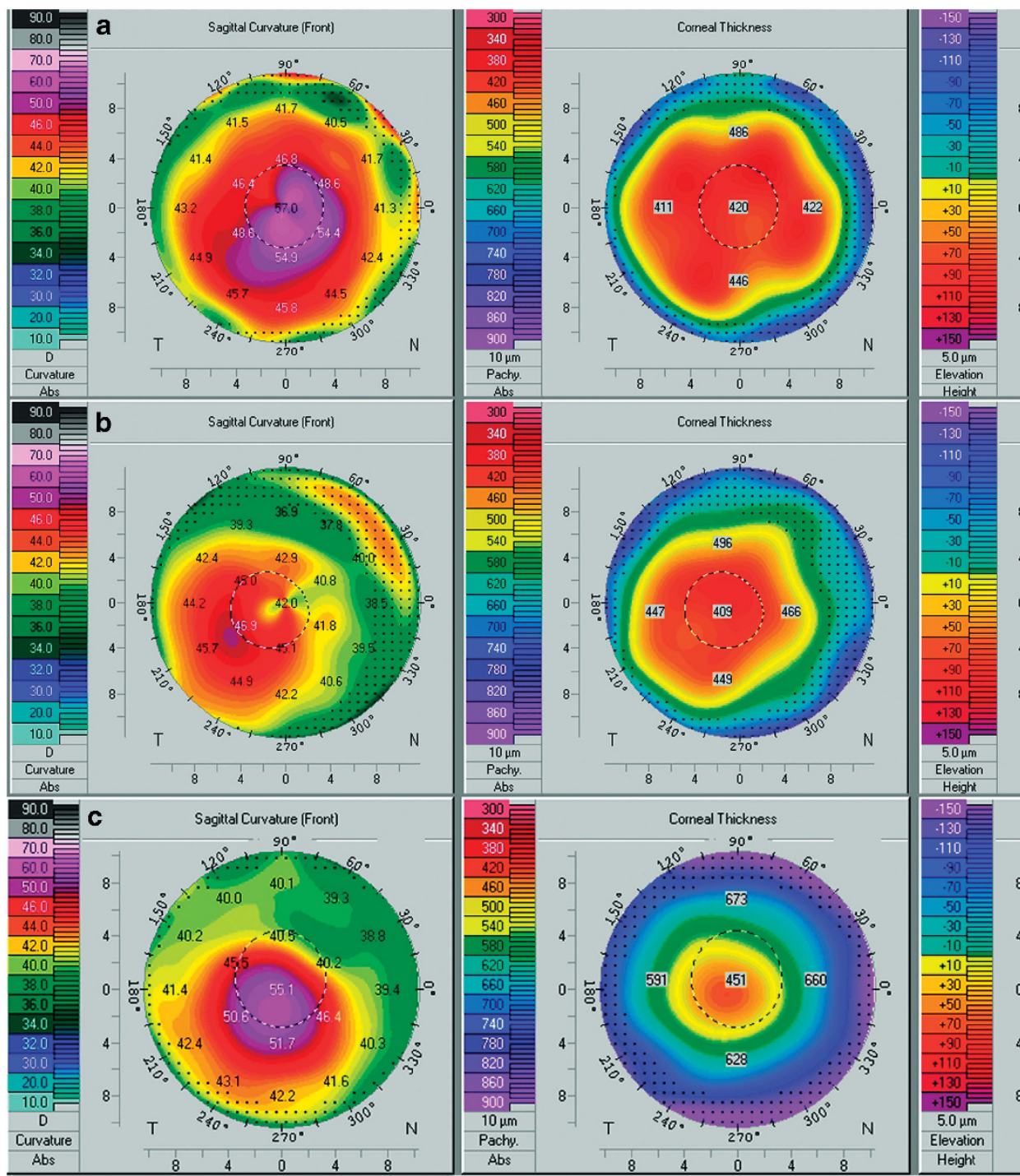

Elevation (Back) BFS=6.32 Float, Dia=8.00
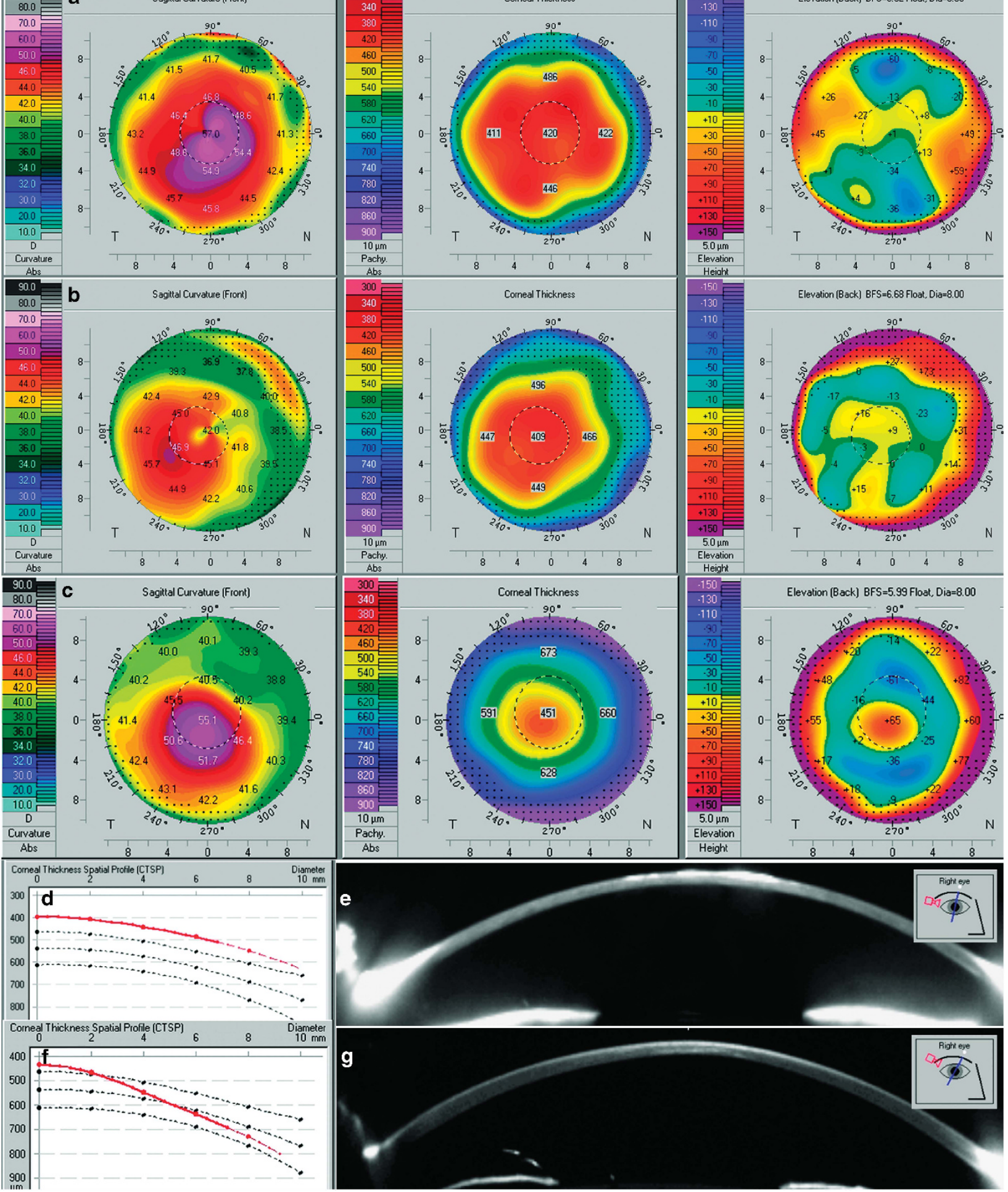

Figure 1 Scheimpflug imaging of macular corneal dystrophy and keratoconus corneas. Anterior sagittal curvature, pachymetry, and posterior elevation maps of the right cornea of proband 1 (a) and the right cornea of proband 5 (b), note diffuse thinning on pachymetry map and lack of posterior elevation in both a and b. Right cornea of a patient with keratoconus, note localized ectasia observed on all three maps (c). Corneal thickness spatial profile of the right cornea of proband 5, note uniformly thin cornea shown by a red line (d). Single Scheimpflug image of the right cornea of proband 1 further documenting diffuse thinning; imbedded image shows meridian of capture (e). Corneal thickness spatial profile of keratoconic cornea, note progressive thinning towards the corneal center (f). Single Scheimpflug image of the right cornea of a keratoconus patient documenting localized paracentral thinning, imbedded image shows meridian of capture (g). 
individual Scheimpflug images (Figure 1e). These observations are in contrast to the focal thinning and posterior corneal elevation seen in the patient with keratoconus (Figures 1c, f and g). Histopathological examination of all three corneas from probands $2-4$ confirmed the presence of corneal thinning together with characteristic glycosaminoglycan deposits that stained positive with Alcian blue, in keratocytes, endothelial cells, and extracellularly in the stroma and Descemet membrane (data not shown).

Two novel CHST6 mutations, c.13C > T; p.(Arg5Cys) and c.289C > T; p.(Arg97Cys), and four previously reported disease-causing mutations were identified (Table 1, Supplementary Material). The novel missense mutations were not found in sequence variant databases. MutPred indicated p.(Arg5Cys) to be possibly harmful with an overall score of $g=0.556$ and Polyphen 2 as damaging scoring 1.000, whereas SNPs\&GO evaluated the mutation as neutral with a reliability index of 8 . All three tools predicted p.(Arg97Cys) to be deleterious (PolyPhen2 scored it 1.000, MutPred 0.789, and SNPs\&GO as disease related with a reliability index of 9). Within the compound allele c.[484C > G; 599T > G], the variant at position 484 is a common polymorphism in linkage disequilibrium with the disease-causing mutation at position $599 .^{8}$

\section{Discussion}

It has been suggested that the concomitant occurrence of keratoconus and various other corneal dystrophies may be higher than would be expected by chance. ${ }^{6}$ Regular corneal astigmatism and corneal thinning are characteristic features of MCD. ${ }^{2}$ Corneal astigmatism and corneal thinning are also features of keratoconus, and in this respect the phenotype of the two conditions is similar. MCD is an autosomal recessive corneal dystrophy with an identified genetic cause; however, the contribution of genetic factors to the development of keratoconus is not known. ${ }^{14}$ Given our current lack of knowledge of the cause of keratoconus, it is important to determine whether there is any potential involvement of the CHST6 gene or associated pathways. Interestingly, concurrent keratoconus and MCD has been previously described in five cases, ${ }^{15-18}$ and one of the linked loci for keratoconus (16q22.3-q23.1) contains the CHST6 gene. ${ }^{19}$

When we used the Pentacam Scheimpflug system to evaluate anterior corneal surface parameters in cases with MCD, there was a pattern suggestive of keratoconus in all six eyes that were examined. However, there was not an associated elevation of the posterior corneal surface. Importantly, in contrast to the changes that characterize keratoconus in which there is localized stromal thinning, corneas with MCD showed diffuse thinning that involved the whole diameter of the cornea. Diffuse corneal thinning was also present on histopathological examination of corneas with MCD compared with control corneal specimens. Therefore evaluation of the anterior corneal surface in isolation can give indices that spuriously suggest the presence of keratoconus, and correlation with posterior corneal elevation maps and regional pachymetry is required. ${ }^{20,21}$ The origin of the apparent anterior corneal elevation is uncertain, but the stromal deposits of MCD probably affect the quality of data capture.

Pathogenic mutations within CHST6 were found in all four probands with MCD, including two novel missense mutations; c.13C > T; p.(Arg5Cys) and c.289C > T; p.(Arg97Cys) were considered disease-causing based on their absence in sequence variant databases and bioinformatic analysis. Over the last 10 years we have examined 17 affected individuals from 12 families with MCD at the Department of Ophthalmology in Prague. The complex allele with a polymorphic missense variant in linkage disequilibrium with the causative mutation c.[484C > G; 599T> G]; p.[Arg162Gly; Leu200Arg] was found in 8 of the 12 families of this Czech MCD cohort, with two homozygous probands and a further six probands who were heterozygous. ${ }^{8}$

In conclusion, our results suggest that the change in anterior corneal curvature and diffuse corneal thinning is a phenocopy of the changes that occur in keratoconus. The apparent ectasia in this cohort of patients with MCD differs in several important aspects from the changes that define keratoconus. Thinning and corneal distortion is to be expected if there is dysregulation of keratan sulfate proteoglycan synthesis or catabolism that influences corneal structure. ${ }^{22}$

\section{Summary}

What was known before

- Corneal thinning is an important clinical feature of macular corneal dystrophy.

- Corneal astigmatism and corneal thinning are also features of keratoconus, and in this respect the phenotype of the two conditions is similar.

What this study adds

- Localized elevation of the anterior corneal curvature can occur in macular corneal dystrophy in the absence of other features that would define a diagnosis of keratoconus.

- The identification of a further two Czech probands with the compound allele c.[484C > G; $599 \mathrm{~T}>\mathrm{G}$ ] supports the enrichment of this allele in the study population.

- Two novel CHST6 mutations were also found. 


\section{Conflict of interest}

The authors declare no conflict of interest.

\section{Acknowledgements}

Institutional support was provided by UNCE 204011 and PRVOUK-P24/LF1/3 program of the Charles University in Prague. LD was supported by the research grant SVV 260022/2014. AJH and SJT are supported by the National Institute for Health Research (NIHR) Biomedical Research Centre at Moorfields Eye Hospital NHS Foundation Trust and the UCL Institute of Ophthalmology.

\section{References}

1 Klintworth GK. Corneal dystrophies. Orphanet J Rare Dis 2009; 4: 7.

2 Donnenfeld ED, Cohen EJ, Ingraham HJ, Poleski SA, Goldsmith E, Laibson PR. Corneal thinning in macular corneal dystrophy. Am J Ophthalmol 1986; 101: 112-113.

3 Akama TO, Nishida K, Nakayama J, Watanabe H, Ozaki K, Nakamura T et al. Macular corneal dystrophy type I and type II are caused by distinct mutations in a new sulphotransferase gene. Nat Genet 2000; 26: 237-241.

4 Ihalainen A. Clinical and epidemiological features of keratoconus genetic and external factors in the pathogenesis of the disease. Acta Ophthalmol Suppl 1986; 178: 1-64.

5 Tuft SJ, Hassan H, George S, Frazer DG, Willoughby CE, Liskova P. Keratoconus in 18 pairs of twins. Acta Ophthalmol 2012; 90: e482-e486.

6 Cremona FA, Ghosheh FR, Rapuano CJ, Eagle Jr RC, Hammersmith KM, Laibson PR et al. Keratoconus associated with other corneal dystrophies. Cornea 2009; 28: 127-135.

7 Lechner J, Dash DP, Muszynska D, Hosseini M, Segev F, George $\mathrm{S}$ et al. Mutational spectrum of the ZEB1 gene in corneal dystrophies supports a genotype-phenotype correlation. Invest Ophthalmol Vis Sci 2013; 54: 3215-3223.

8 Liskova P, Veraitch B, Jirsova K, Filipec M, Neuwirth A, Ebenezer ND et al. Sequencing of the CHST6 gene in Czech macular corneal dystrophy patients supports the evidence of a founder mutation. Br J Ophthalmol 2008; 92: 265-267.

9 Liskova P, Hysi PG, Williams D, Ainsworth JR, Shah S, de la Chapelle A et al. Study of p.N247S KERA mutation in a British family with cornea plana. Mol Vis 2007; 13: 1339-1347.

10 den Dunnen JT, Antonarakis SE. Mutation nomenclature extensions and suggestions to describe complex mutations: a discussion. Hum Mutat 2000; 15: 7-12.

11 Adzhubei IA, Schmidt S, Peshkin L, Ramensky VE, Gerasimova A, Bork P et al. A method and server for predicting damaging missense mutations. Nat Methods 2010; 7: $248-249$.

12 Li B, Krishnan VG, Mort ME, Xin F, Kamati KK, Cooper DN et al. Automated inference of molecular mechanisms of disease from amino acid substitutions. Bioinformatics 2009; 25: 2744-2750.

13 Calabrese R, Capriotti E, Fariselli P, Martelli PL, Casadio R. Functional annotations improve the predictive score of human disease-related mutations in proteins. Hum Mutat 2009; 30: 1237-1244.

14 Burdon KP, Vincent AL. Insights into keratoconus from a genetic perspective. Clin Exp Optom 2013; 96: 146-154.

15 Javadi MA, Rafee'i AB, Kamalian N, Karimian F, Ja'farinasab MR, Yazdani S. Concomitant keratoconus and macular corneal dystrophy. Cornea 2004; 23: 508-512.

16 Balestrazzi A, Martone G, Traversi C, Haka G, Toti P, Caporossi A. Keratoconus associated with corneal macular dystrophy: in vivo confocal microscopic evaluation. Eur J Ophthalmol 2006; 16: 745-750.

17 Al-Hamdan G, Al-Mutairi S, Al-Adwani E, Al-Mujaini A. Bilateral coexistence of keratoconus and macular corneal dystrophy. Oman J Ophthalmol 2009; 2: 79-81.

18 Mohammad-Rabei H, Shojaei A, Aslani M. Concurrent macular corneal dystrophy and keratoconus. Middle East Afr J Ophthalmol 2012; 19: 251-253.

19 Tyynismaa H, Sistonen P, Tuupanen S, Tervo T, Dammert A, Latvala $\mathrm{T}$ et al. A locus for autosomal dominant keratoconus: linkage to 16q22.3-q23.1 in Finnish families. Invest Ophthalmol Vis Sci 2002; 43: 3160-3164.

20 Tomidokoro A, Oshika T, Amano S, Higaki S, Maeda N, Miyata K. Changes in anterior and posterior corneal curvatures in keratoconus. Ophthalmology 2000; 107: 1328-1332.

21 Schlegel Z, Hoang-Xuan T, Gatinel D. Comparison of and correlation between anterior and posterior corneal elevation maps in normal eyes and keratoconus-suspect eyes. J Cataract Refract Surg 2008; 34: 789-795.

22 Akhtar S, Bron AJ, Hayes AJ, Meek KM, Caterson B. Role of keratan sulphate (sulphated poly -N-acetyllactosamine repeats) in keratoconic cornea, histochemical, and ultrastructural analysis. Graefes Arch Clin Exp Ophthalmol 2011; 249: 413-420.

Supplementary Information accompanies this paper on Eye website (http://www.nature.com/eye) 